\title{
Compact dimensions and the Casimir effect: the Proca connection
}

\author{
${ }^{1,2}$ Ariel Edery* \\ ${ }^{1}$ Physics Department, Bishop's University \\ 2600 College Street, Sherbrooke, Québec, Canada J1M 0C8 \\ ${ }^{2 \dagger}$ Kavli Institute for Theoretical Physics, University of California \\ Kohn Hall, Santa Barbara, CA 93106 USA \\ ${ }^{3,4}$ Valery N. Marachevsky ${ }^{\ddagger}$ \\ ${ }^{3}$ Laboratoire Kastler Brossel, CNRS, ENS, UPMC, \\ Campus Jussieu case 74, 75252 Paris, France \\ ${ }^{4}$ V. A Fock Institute of Physics, St. Petersburg State University \\ 198504 St. Petersburg, Russia
}

\begin{abstract}
We study the Casimir effect in the presence of an extra dimension compactified on a circle of radius $R$ ( $M^{4} \times S^{1}$ spacetime). Our starting point is the Kaluza Klein decomposition of the $5 D$ Maxwell action into a massless sector containing the $4 D$ Maxwell action and an extra massless scalar field and a Proca sector containing $4 D$ gauge fields with masses $m_{n}=n / R$ where $n$ is a positive integer. An important point is that, in the presence of perfectly conducting parallel plates, the three degrees of freedom do not yield three discrete (non-penetrating) modes but two discrete modes and one continuum (penetrating) mode. The massless sector reproduces Casimir's original result and the Proca sector yields the corrections. The contribution from the Proca continuum mode is obtained within the framework of Lifshitz theory for plane parallel dielectrics whereas the discrete modes are calculated via $5 D$ formulas for the piston geometry. An interesting manifestation of the extra compact dimension is that the Casimir force between perfectly conducting plates depends on the thicknesses of the slabs.
\end{abstract}

\footnotetext{
*Email: aedery@ubishops.ca

${ }^{\dagger}$ work partly completed at KITP, Santa Barbara

${ }^{\ddagger}$ Email: maraval@mail.ru
} 


\section{Introduction}

In the 1920's, Kaluza [1] and Klein [2] attempted to unify classical gravitation and electromagnetism by extending General Relativity to a five-dimensional $\left(M^{4} \times S^{1}\right)$ spacetime. In modern times, string theory revived the idea of extra dimensions from a more fundamental perspective. In this paper, we are interested in the effect of a compact dimension on Casimir's original parallel plate scenario. Before we discuss this subject, we note that the combined effect of compact dimensions and Casimir energies has recently attracted interest in cosmology [3, 4]. For example, in a brane world scenario with toroidal extra dimensions, it was found that Casimir energies can play a central role in attempting to resolve some long outstanding puzzles. It was shown that under certain conditions, Casimir energies can stabilize the extra dimensions, allow three dimensions to grow large and provide an effective dark energy in the large dimensions [3]. Most recently, there has been some interesting work on Casimir energies in Randall Sundrum models [5, 6] and in one-dimensional piston scenarios with extra compact dimensions for a massless scalar field obeying Dirichlet boundary conditions [7]. The UV cutoff dependence of the Casimir energy for perfect conductors with an extra compact dimension was also studied recently in [8] (the compactification scheme is not explicitly stated). Closer to the spirit of our work, the perfectly conducting parallel plate scenario with an extra dimension compactified to an $S^{1} / Z_{2}$ orbifold was studied in [9] and explicit analytic expressions for the Casimir force were obtained.

In this paper, we calculate the correction to the Casimir force due to an extra dimension compactified on a circle of radius $R$ for the case of perfectly conducting parallel plates separated by a distance $a$. Our starting point in Sec. 2 is the Kaluza Klein (KK) decomposition of the $4+1$ $(5 D)$ Maxwell action into two sectors in $4 D$ : a massless and massive (Proca) sector [10]. The massless sector contains the $4 D$ Maxwell action as well as a $4 D$ massless scalar field. The Proca

sector yields an infinite set of $4 \mathrm{D}$ massive gauge fields $A_{\mu}^{(n)}$ (and $A_{\mu}^{(n) *}$ ) with masses $m_{n}=n / R$ where $n$ is a positive integer and $R$ is the radius of the compact dimension. An advantage of the $K K$ decomposition is that the problem can now be analyzed in four spacetime dimensions without reference to the extra fifth compact dimension. In $5 D$, the photon has three degrees of freedom or polarizations and the $K K$ decomposition must yield the same number of degrees of freedom since it describes the same physical system. In the massless sector, the $4 D$ Maxwell term yields the usual two polarizations and the $4 D$ massless scalar field yields one degree of freedom for a total of three. In the $4 D$ Proca sector, it is well known that the massive photon has three polarizations because of the presence of a longitudinal mode in addition to the usual two transverse modes. Both sectors have three degrees of freedom as in the original $5 D$ case.

In Sec. 3 the mode decomposition is explained in detail. An important point is that in the presence of perfectly conducting plates, not all three modes reflect perfectly at the boundary and one does not obtain three discrete (non-penetrating) modes. The three polarizations yield 
two discrete and one continuum (penetrating) mode. This point does not seem to have been taken into account in previous work [8, 9]. In $4 D$ Proca theory, unlike the usual Maxwell theory in $4 D$, there exists a propagating continuum mode inside the perfect conductors [11]. The electric and magnetic fields inside the perfect conductors are zero but the gauge potentials are non-zero contributing a non-zero energy density given by $m^{2}\left(\left(A^{0}\right)^{2}+\mathbf{A}^{2}\right)$ where $m$ is the mass of the photon. The penetrating continuum mode requires analyzing only the third component $A_{z}$ of the gauge potential [11. The boundary conditions on $A_{z}$ and its derivative $\partial_{z} A_{z}$ are that they be continuous at the boundaries and this is equivalent to the boundary conditions on a transverse-electric (TE) mode propagating in plane parallel dielectrics of different permittivities. The Casimir contribution from the continuum mode can therefore be calculated efficiently by making use of Lifshitz theory [12].

The massless sector reproduces exactly Casimir's original result [13]. The Proca sector is responsible for the corrections due to the extra compact dimension and these are derived in Sec. 4. The corrections from the discrete modes are a function of the radius $R$ of the compact dimension and the correction from the continuum mode is a function of both the radius $R$ and the thicknesses $\ell_{1}$ and $\ell_{2}$ of the two perfectly conducting slabs respectively. In the limit as $R \rightarrow 0$ both the Proca discrete and continuum mode corrections vanish and one recovers Casimir's original result.

Casimir force calculations for the Proca discrete and the Proca continuum modes are fundamentally different. As already mentioned, the continuum modes are calculated via Lifshitz theory. For the discrete modes we derive in the appendix Casimir piston formulas for Dirichlet boundary conditions that are a $5 D$ generalization of previously derived $4 D$ expressions [14. We then make use of the parallel plate limit of these formulas. The advantage of the piston scenario [15] is that it automatically includes the Casimir contribution from the exterior region. The piston separates the volume into an interior and exterior and the main point is that contributions from both chambers must be included in any realistic calculation of the Casimir force. Some of the first exact results in $3+1$ dimensions include the $3+1$ Dirichlet piston [16] and the 3+1 EM piston [14, 17] (see also refs. [18]-[27] with a review in [18]).

We plot the contribution from both the Proca discrete and continuum modes as a function of the circumference $d=2 \pi R$. The discrete modes make a $12.1 \%$ correction to Casimir's original result when $d=2$ (lengths are in units of the plate separation $a$ ). The continuum mode yields a correction of $0.6 \%$ at $d=2$ when the thickness of each slab is $\ell_{1}=10$ (this is a maximum at $d=2$ since $\ell_{1}=10$ yields results that are almost identical to $\ell_{1}=\infty$ ). The total correction to Casimir's result at $d=2$ and $\ell_{1}=10$ is therefore $12.7 \%$ (the maximum correction at $d=2$ ). Both corrections decrease exponentially fast as $d$ decreases reaching less than $1 \%$ at $d=1$.

In Sec. 5 we summarize our results and discuss some relevant high-precision Casimir experiments that may be important in the future to detect the effect of extra dimensions. 
We use units with $\hbar=c=1$ throughout.

\section{Kaluza Klein decomposition of the $5 D$ Maxwell action}

This section follows closely the TASI lectures, "To the Fifth dimension and Back" by Raman Sundrum [10] (the signature is $(+,-,-,-,-)$ ). We will therefore be brief but explain enough for the work to be self-contained and applicable to our particular case (i.e. abelian gauge fields). The Maxwell action in $5 D$ is given by

$$
\begin{aligned}
S & =\int d^{4} x \int d x^{4}\left\{-\frac{1}{4} F_{a b} F^{a b}\right\} \\
& =\int d^{4} x \int d x^{4}\left\{-\frac{1}{4} F_{\mu \nu} F^{\mu \nu}-\frac{1}{2} F_{\mu 4} F^{\mu 4}\right\}
\end{aligned}
$$

where $a$ and $b$ are $5 D$ indices (they run from 0 to 4 inclusively) and $\mu$ and $\nu$ are $4 D$ indices (they run from 0 to 3 inclusively). If the fourth spatial dimension is compactified to a circle of radius $R$ we can express $x^{4}$ as $R \phi$ where $\phi$ is an angular coordinate $-\pi \leq \phi \leq \pi$. We then can Fourier expand the (abelian) gauge fields as

$$
A_{b}\left(x^{\mu}, \phi\right)=A_{b}^{(0)}(x)+\sum_{n=1}^{\infty}\left(A_{b}^{(n)}(x) e^{i n \phi}+\text { c.c. }\right)
$$

An important point is that it is not possible to go to axial gauge $A_{4}=0$ because it is not possible to remove the " $\mathrm{n}=0$ " part $A_{4}^{(0)}$. This would require a gauge transformation with function $\Lambda=-x^{4} A_{4}^{(0)}$ so that $\partial_{4} \Lambda=-A_{4}^{(0)}$. However, such a $\Lambda$ is not valid because it is proportional to $x^{4}$ and hence is not periodic. As pointed out in [10], the closest to axial gauge one can reach is "almost axial" gauge where $A_{4}$ does not depend on $x^{4}$ i.e. $A_{4}(x, \phi)=A_{4}^{(0)}(x)$. The action (2.2) then becomes [10]

$$
\begin{aligned}
S= & \int d^{4} x \int_{-\pi}^{\pi} R d \phi\left\{-\frac{1}{4} F_{\mu \nu} F^{\mu \nu}+\frac{1}{2}\left(\partial_{\mu} A_{4}^{(0)}\right)^{2}+\frac{1}{2}\left(\partial_{4} A_{\mu}\right)^{2}\right\} \\
=2 & \pi R \int d^{4} x\left\{-\frac{1}{4} F_{\mu \nu}^{(0)} F^{\mu \nu(0)}+\frac{1}{2}\left(\partial_{\mu} A_{4}^{(0)}\right)^{2}\right. \\
& \left.+\sum_{n=1}^{\infty}\left[-\frac{1}{2}\left|\partial_{\mu} A_{\nu}^{(n)}-\partial_{\nu} A_{\mu}^{(n)}\right|^{2}+\frac{n^{2}}{R^{2}}\left|A_{\mu}^{(n)}\right|^{2}\right]\right\} .
\end{aligned}
$$

The 5D Maxwell action decomposes into a $4 D$ massless sector containing $4 D$ Maxwell plus an extra scalar field $A_{4}^{0}$ and a Proca sector with an infinite set of $4 D$ gauge fields $A_{\mu}^{(n)}$ (and $A_{\mu}^{(n) *}$ ) of mass $m_{n}=n / R$ where $R$ is the radius of the compact dimension and $n$ is a positive integer. 


\section{Mode decomposition in the presence of perfectly conducting parallel plates}

\subsection{Massless Sector}

We now find the mode decomposition in the presence of perfectly conducting parallel plates separated by a distance $a$ (situated at $z=0$ and $z=a$ ). We begin with the massless sector and then look at the Proca sector. In the massless sector, we have the usual four gauge components $A_{0}^{(0)}, A_{1}^{(0)}, A_{2}^{(0)}$ and $A_{3}^{(0)}$ of $4 \mathrm{D}$ Maxwell plus an extra 4D massless scalar field $\phi \equiv A_{4}^{(0)}$. This yields three degrees of freedom or polarizations: the usual two polarizations from $4 D$ Maxwell and one extra degree of freedom from the massless scalar field $\phi$. We can go to radiation gauge where $A_{0}^{(0)}=0$ and $\partial_{i} A^{i^{(0)}}=0$ where $i=1,2,3$ (this can be extended to include $i=4$ since $\partial_{4} A^{4^{(0)}}$ is identically zero). At the surface of the plates the electric field components $E_{1}$ and $E_{2}$ are zero so that $A_{1}^{(0)}$ and $A_{2}^{(0)}$ are zero at the surface of the conducting plates. The gauge condition $\partial_{i} A^{i^{(0)}}=0$ yields a condition on $A_{3}^{(0)}$, namely $\partial_{3} A^{3^{(0)}}=0$ at the surface of the plates. The field $\phi$ is a continuum mode that obeys the wave equation $\square \phi=0$ for a free massless scalar field. The perfect conductors do not impose any extra condition on the scalar field. The mode decomposition for the massless sector is then given by

$$
\left.\begin{array}{rl}
A_{0}^{(0)} & =0 \\
A_{1}^{(0)} & =c_{1} \sin \left(k_{z} z\right) e^{i(\mathbf{k} \cdot \mathbf{x}-\omega t)} \\
A_{2}^{(0)} & =c_{2} \sin \left(k_{z} z\right) e^{i(\mathbf{k} \cdot \mathbf{x}-\omega t)} \\
A_{3}^{(0)} & =c_{3} \cos \left(k_{z} z\right) e^{i(\mathbf{k} \cdot \mathbf{x}-\omega t)} \\
A_{4}^{(0)} & =c_{4} e^{i\left(\mathbf{k} \cdot \mathbf{x}+p_{z} z-\omega^{\prime} t\right)}
\end{array}\right\} \begin{aligned}
k_{z} & =\frac{n \pi}{a} \quad n=1,2,3 \ldots \\
\mathbf{k} & =\left(k_{x}, k_{y}\right) \\
\mathbf{x} & =(x, y) \\
\omega^{2} & =\mathbf{k}^{2}+k_{z}^{2}=\mathbf{k}^{2}+\frac{n^{2} \pi^{2}}{a^{2}}
\end{aligned}
$$

where the momenta $p_{z}$ and $k_{x}, k_{y}$ are continuous. The three modes $A_{1}^{(0)}, A_{2}^{(0)}$ and $A_{3}^{(0)}$ yield two independent modes because of the gauge condition $\partial_{i} A^{i}=0$. These two modes are discrete containing the quantized momentum $k_{z}=n \pi / a$. The third independent mode is the massless scalar field $\phi$ and it is a continuum mode. The important point is that there are three independent modes but only two are discrete. The scalar field $\phi$ makes no contribution to the Casimir force between the plates because it is a free field throughout the spacetime: it is not influenced by the perfectly conducting boundary conditions imposed on the electromagnetic field in $4 D$. The two discrete modes and their frequencies $\omega$ are identical to what appears in the usual 4D case with perfectly conducting parallel plates. They therefore reproduce exactly Casimir's result and we can simply quote the result. The Casimir force per unit area (the 
pressure $\left.P_{0}\right)$ stemming from the massless sector is given by [13]

$$
P_{0}=-\frac{\pi^{2}}{240 a^{4}}
$$

\subsection{Proca sector: two discrete modes and one continuum mode}

The Casimir effect for photons of mass $m$ (Proca theory) in the presence of perfectly conducting plates was analyzed in detail by Barton and Dombey [11. In the presence of perfectly conducting parallel plates, Barton and Dombey showed that the three polarizations in Proca theory yield two discrete modes and one continuum mode. The important point is that the three polarizations do not yield three but two discrete modes. The discrete modes are perfectly reflected or non-penetrating modes where the gauge fields $A_{\mu}$ are zero both inside and on the surface of the conductor [11]. The latter condition (i.e. gauge potentials are zero on the surface) stems from the continuity of the gauge potentials in Proca theory. The contribution of the two discrete modes does not depend on the thickness of the conductors and yields an $R$-dependent correction to Casimir's original parallel plate result $P_{0}$ given by (3.7). The continuum mode penetrates through the conductors and makes a contribution to the Casimir force that depends on the thicknesses $\ell_{1}$ and $\ell_{2}$ of the two conducting slabs and the radius $R$ of the compact dimension. This contribution enters into the Casimir force in a fundamentally different way than the discrete modes. Both the continuum and discrete mode corrections vanish in the limit $R \rightarrow 0$.

\subsubsection{Discrete modes}

With the Lorentz condition $\partial^{\mu} A_{\mu}^{(n)}=0$, the equations of motion outside the conductor are given by $\left(\square+m_{n}^{2}\right) A_{\mu}^{(n)}=0$ where $m_{n}^{2}=n^{2} / R^{2}$. For the discrete modes, the perfect conductor boundary conditions are that the gauge components $A_{0}^{(n)}, A_{1}^{(n)}, A_{2}^{(n)}$ and $A_{3}^{(n)}$ are zero on the surface of the conductor (at $z=0$ and $z=a$ ). The mode decomposition for the discrete modes is given by [1]

$$
\left.\begin{array}{l}
A_{0}^{(n)}=c_{0} \sin \left(k_{z} z\right) e^{i(\mathbf{k} \cdot \mathbf{x}-\omega t)} \\
A_{1}^{(n)}=c_{1} \sin \left(k_{z} z\right) e^{i(\mathbf{k} \cdot \mathbf{x}-\omega t)} \\
A_{2}^{(n)}=c_{2} \sin \left(k_{z} z\right) e^{i(\mathbf{k} \cdot \mathbf{x}-\omega t)} \\
A_{3}^{(n)}=0
\end{array}\right\} \begin{gathered}
k_{z}=\frac{\ell \pi}{a} \quad(\ell=1,2, . .) \\
\mathbf{k}=\left(k_{x}, k_{y}\right) ; \mathbf{x}=(x, y) \\
\omega^{2}=\mathbf{k}^{2}+k_{z}^{2}+m_{n}^{2}
\end{gathered}
$$

Note that $A_{3}^{(n)}$ cannot be a discrete mode (either a $\cos \left(k_{z} z\right)$ or $\sin \left(k_{z} z\right)$ term) because $A_{3}^{(n)}$ must be both zero on the surface of the conductor and satisfy the Lorentz condition. The three 
modes $A_{0}^{(n)}, A_{1}^{(n)}$ and $A_{2}^{(n)}$ together with the Lorentz condition yield two independent discrete modes for every $n$. These have frequency $\omega$ given by

$$
\omega^{2}=\mathbf{k}^{2}+k_{z}^{2}+m_{n}^{2}=k_{x}^{2}+k_{y}^{2}+\frac{\ell^{2} \pi^{2}}{a^{2}}+\frac{n^{2}}{R^{2}} .
$$

where $\ell$ and $n$ are both positive integers that run from 1 to $\infty$. The same analysis applies to the fields $A_{\mu}^{(n) *}$. The parallel plate geometry can be thought of as a rectangular box with plate separation $a$ and plate area $b \times c$ where $b$ and $c$ are taken to be large (infinite limit). The continuous momenta $k_{x}$ and $k_{y}$ can be expressed as $n_{x} \pi / b$ and $n_{y} \pi / c$ in the limit $b, c \rightarrow \infty$, with $n_{x}$ and $n_{y}$ positive integers. The frequency is then given by

$$
\omega=\frac{\ell^{2} \pi^{2}}{a^{2}}+\frac{n_{x}^{2} \pi^{2}}{b^{2}}+\frac{n_{y}^{2} \pi^{2}}{c^{2}}+\frac{n^{2}}{R^{2}} \text { where } b \text { and } c \text { are assumed large (infinite limit) . }
$$

Let $E_{D}$ be the $4+1$ dimensional Dirichlet Casimir energy defined by the sum over the four quantum numbers $\ell, n_{x}, n_{y}$ and $n$ of $\omega / 2$ from 1 to $\infty$ (i.e. each of the four sums starts at 1 ). The Casimir energy for the Proca discrete modes $E_{p r d}$ is then equal to four times the Dirichlet Casimir energy $E_{D}$ :

$$
E_{p r d}=4 E_{D}
$$

The factor of four stems from the Proca sector having two discrete modes for $A_{\mu}^{(n)}$ and two discrete modes for $A_{\mu}^{(n) *}$.

\subsubsection{Continuum mode and the equivalence with TE mode in dielectric}

Besides the two discrete modes, Proca theory in the presence of conducting plates has a continuum (penetrating) mode which also contributes to the Casimir energy. This has been analyzed in [11]. In our case of an extra compact dimension we replace the mass $m$ in Proca theory by the mass $m_{n}=n / R$ (we then sum over all $n$ when calculating the Casimir energy).

The continuum mode requires analyzing only the component $A_{z}$ [11] (in our case we have $A_{z}^{(n)}$ for each $n$ ). In the Proca theory vector potentials of the electromagnetic field are continuous and satisfy the Lorentz condition. The latter implies that $\partial_{z} A_{z}^{(n)}$ is continuous across the boundary since $\partial_{t} A_{0}^{(n)}$ and $\nabla_{\|} \mathbf{A}_{\|}^{(n)}$ are continuous across the boundary. Therefore in Proca theory the field $A_{z}^{(n)}$ and its normal derivative $\partial_{z} A_{z}^{(n)}$ are continuous across the flat boundaries perpendicular to the $z$ direction [11].

The electric field is zero inside the perfect conductors. From this condition and the Lorentz condition it follows that the $A_{z}^{(n)}$ components satisfy the equation for a massless scalar field inside the conductors $\square A_{z}^{(n)}=0$. Outside the media (in vacuum) the $A_{z}^{(n)}$ components satisfy 


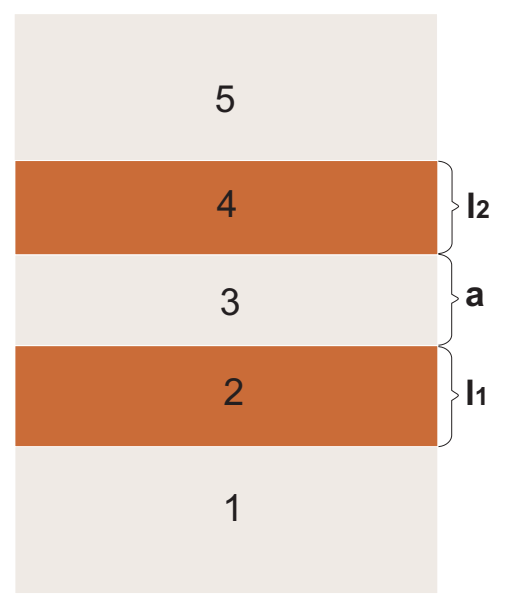

Figure 1: The parallel plate set-up with its five regions. The five regions starting with region 1 are: exterior (vacuum), conducting slab of thickness $\ell_{1}$, vacuum gap of length $a$, conducting slab of thickness $\ell_{2}$, exterior (vacuum).

the equation for a massive scalar field $\left(\square+m_{n}^{2}\right) A_{z}^{(n)}=0$. In the parallel plate set-up there are five regions (see Fig.1). Region 1 is the exterior vacuum, region 2 is a conducting slab of thickness $\ell_{1}$, region 3 is the gap of length $a$ (in vacuum), region 4 is a conducting slab of thickness $\ell_{2}$ and region 5 is the exterior vacuum. Let $m$ and $m=0$ correspond to massive and massless propagation. From region 1 to region 5 , we therefore have the sequence $m, m=$ $0, m, m=0, m$.

The equations of motion for $A_{z}^{(n)}$ and the conditions of continuity for $A_{z}^{(n)}$ and its derivative $\partial_{z} A_{z}^{(n)}$ are exactly equivalent to the conditions for the transverse electric (TE) electromagnetic mode propagating in slabs of different dielectric permittivities. Massive propagation is equivalent to TE propagation in a dielectric with permittivity $\epsilon(\omega)=1-\omega_{p}^{2} / \omega^{2}$ where the plasma frequency is given by $\omega_{p}=m_{n}$. Massless propagation is equivalent to propagation in a dielectric with permittivity $\epsilon=1$. The conditions of continuity for the TE mode at the boundaries are the same as those on $A_{z}^{(n)}$ and its derivative. Therefore if the five regions are replaced by dielectrics with permittivities $\epsilon(\omega), \epsilon=1, \epsilon(\omega), \epsilon=1, \epsilon(\omega)$ corresponding to the sequence $m, m=0, m, m=0, m$ we obtain a physically equivalent set-up. The upshot of all this is that the Casimir energy of the continuum mode $A_{z}^{(n)}$ can be calculated in the framework of Lifshitz theory.

The same conclusions can be applied to the continuum modes $A_{z}^{(n) *}$ and they make the same contribution to the Casimir energy as $A_{z}^{(n)}$. 


\section{Corrections to the Casimir force}

\subsection{Proca discrete modes}

The Dirichlet Casimir energy $E_{D}$ in a piston geometry associated with the frequency $\omega$ is given by (see appendix)

$$
E_{D}=-\frac{1}{2 \pi} \sum_{k m n=1}^{\infty} \sum_{\ell=1}^{\infty} \frac{\lambda_{k m n}}{\ell} K_{1}\left(2 \ell \lambda_{k m n} a\right) \text { with } \lambda_{k m n}=\sqrt{(\pi k / b)^{2}+(\pi m / c)^{2}+(n / R)^{2}}
$$

The sum $\sum_{k m n=1}^{\infty}$ is short-hand for a triple sum with each sum running from 1 to $\infty$. The expression (4.11) for the Dirichlet energy in a piston geometry automatically includes the contribution from both the interior and exterior regions of the plates. The Casimir energy due to Proca discrete modes is given by $E_{p r d}=4 E_{D}$. The Casimir force due to Proca discrete modes is given by the negative derivative with respect to the plate separation $a$ :

$$
\begin{aligned}
F_{p r d} & =-\frac{\partial}{\partial a} E_{p r d} \\
& =-\frac{2}{\pi} \sum_{k m n=1}^{\infty} \sum_{\ell=1}^{\infty}\left\{\frac{\lambda_{k m n}}{a \ell} K_{1}\left(2 \ell \lambda_{k m n} a\right)+2 \lambda_{k m n}^{2} K_{0}\left(2 \ell \lambda_{k m n} a\right)\right\}
\end{aligned}
$$

The force per unit area is given by $F_{p r d} /(b c)$ in the limit $b, c \rightarrow \infty$. The sums over $k$ and $m$ in (4.12) are therefore replaced by integrals which can be expressed in terms of modified Bessel functions:

$$
\begin{array}{lrl}
\sum_{k=1}^{\infty} \sum_{m=1}^{\infty} \lambda_{k m n} K_{1}\left(2 \ell \lambda_{k m n} a\right) & \sum_{k=1}^{\infty} \sum_{m=1}^{\infty} \lambda_{k m n}^{2} K_{0}\left(2 \ell \lambda_{k m n} a\right) \\
\rightarrow \frac{b c}{2 \pi} \int_{0}^{\infty} \sqrt{r^{2}+\lambda_{n}^{2}} K_{1}\left(2 \ell a \sqrt{r^{2}+\lambda_{n}^{2}}\right) r d r & \rightarrow \frac{b c}{2 \pi} \int_{0}^{\infty}\left(r^{2}+\lambda_{n}^{2}\right) K_{0}\left(2 \ell a \sqrt{r^{2}+\lambda_{n}^{2}}\right) r d r \\
=\left(\frac{b c}{2 \pi}\right)\left(\frac{1}{2 \ell a}\right) \lambda_{n}^{2} K_{2}\left(2 \ell a \lambda_{n}\right) . & =\left(\frac{b c}{4 \pi}\right)\left(\frac{1}{\ell^{2} a^{2}}\right) \lambda_{n}^{2} K_{2}\left(2 \ell a \lambda_{n}\right) \\
& +\left(\frac{b c}{4 \pi}\right)\left(\frac{1}{\ell a}\right) \lambda_{n} K_{1}\left(2 \ell a \lambda_{n}\right) .
\end{array}
$$

After substituting the above in (4.12) with $\lambda_{n}=n / R$, the force per unit area due to the Proca discrete modes is equal to

$$
P_{p r d}=\lim _{b, c \rightarrow \infty} \frac{F_{p r d}}{b c}=-\sum_{n=1}^{\infty} \sum_{\ell=1}^{\infty}\left[\frac{3}{2} \frac{n^{2}}{\pi^{2} R^{2} a^{2} \ell^{2}} K_{2}(2 \ell n a / R)+\frac{n^{3}}{\ell \pi^{2} R^{3} a} K_{1}(2 \ell n a / R)\right] .
$$


The correction coming from the Proca discrete modes in units of Casimir's parallel plate result $P_{0}$ is

$$
\frac{P_{p r d}}{P_{0}}=\sum_{n=1}^{\infty} \sum_{\ell=1}^{\infty}\left[\frac{360}{\pi^{4}} \frac{n^{2}}{\ell^{2}}\left(\frac{a^{2}}{R^{2}}\right) K_{2}(2 \ell n a / R)+\frac{240}{\pi^{4}} \frac{n^{3}}{\ell} \frac{a^{3}}{R^{3}} K_{1}(2 \ell n a / R)\right] .
$$

In Fig. 2, we plot (4.15) as a function of the circumference $d=2 \pi R$ in units of the plate separation $a$. The correction is $12.1 \%$ of Casimir's result when $d=2$ (or $R=1 / \pi$ ) but decreases rapidly (exponentially) as $d$ decreases. It is $2.7 \%$ when $d=1.5,0.098 \%$ when $d=1$ and $16 \times 10^{-7} \%$ when $d=0.5$.

When compact dimensions are not present, the piston geometry does not modify Casimir's parallel plate result in the limit of infinitely sized plates. In this limit the exterior contribution to the Casimir energy is equal to a regularized volume term of an exterior region, which is equal to zero in the zeta function regularization or should be subtracted in other regularizations. However, when compact dimensions are present, the exterior region makes a non-zero contribution even for the case of infinitely sized parallel plates. Therefore, to calculate correctly the effect of a compact dimension on perfectly conducting parallel plates, one must include not only the correction due to the interior but also the correction due to the exterior in all regularizations. The correction (4.14) includes a significant contribution from the exterior. The relevance of the piston geometry is therefore highlighted by the presence of compact dimensions. As an illustration, for the case of $a=1$ and $R=1 / \pi$ (or $d=2$ ), (4.14) yields

$$
P_{\text {prd }}=-0.004990022267 \quad \text { when } R=1 / \pi \text {. }
$$

To determine the interior and exterior contributions to this result we can use formulas for the Dirichlet Casimir piston force $F_{D}$ derived in [20] and then evaluate $F_{p r d}=4 F_{D}$. The Dirichlet piston force $F_{D}$ includes a contribution from the interior $\left(F_{D_{I}}\right)$ and exterior $\left(F_{D_{I I}}\right)$. The interior and exterior contributions can be obtained from equations (3.15) and (3.18) in [20] respectively. We do not explicitly write them out here but simply evaluate them when $R=1 / \pi$ :

$$
\begin{aligned}
\lim _{b, c \rightarrow \infty} \frac{F_{D_{I}}}{b c} & =\frac{\pi}{2^{5}}\left(3 \Gamma(2) \pi^{-3} \zeta(4)-4 \Gamma(5 / 2) \pi^{-7 / 2} \zeta(5)+8 \sum_{n=1}^{\infty} \sum_{\ell=1}^{\infty} \frac{n^{3}}{\ell} K_{1}(2 \pi n \ell)\right) \\
& =0.00121490257 . \\
\lim _{b, c \rightarrow \infty} \frac{F_{D_{I I}}}{b c} & =-\frac{\pi}{2^{5}} \Gamma(5 / 2) \pi^{-7 / 2} \zeta(5)=-0.00246240814 .
\end{aligned}
$$

We therefore obtain

$$
\lim _{b, c \rightarrow \infty} \frac{F_{p r d}}{b c}=4(0.00121490257-0.00246240814)=-0.004990022267
$$


which is in agreement with (4.16).

The important point is that the exterior contribution $F_{D_{I I}}$ is not negligible and in fact, for the case we considered has a higher magnitude then the interior $F_{D_{I}}$. Moreover, note that $F_{D_{I}}$ here is positive and it is only the total $F_{D}=F_{D_{I}}+F_{D_{I I}}$ which is negative (as it must be since $F_{p r d}$ given by (4.12) is manifestly negative). Without the exterior contribution one would erroneously conclude that there is a repulsive force.

\subsection{Proca continuum mode}

As already explained, the Casimir energy due to the continuum mode can be calculated via Lifshitz theory [12, 28, 29, 30]. We assume that the five regions in Fig. 1 are filled with dielectrics of permittivities (starting with region 1) $\epsilon(\omega), \epsilon=1, \epsilon(\omega), \epsilon=1, \epsilon(\omega)$ where $\epsilon(\omega)=$ $1-\omega_{p}^{2} / \omega^{2}$ with the plasma frequency given by $\omega_{p}=m$. The Casimir energy of a TE mode propagating in the dielectrics is then equivalent to the Casimir energy of a Proca continuum mode of mass $m$ (we will later replace $m$ by $m_{n}=n / R$ ). The Casimir energy $E_{c}$ for the continuum is thus given by ( $S$ is the surface of the plates):

$$
E_{c}\left(l_{1}, a, l_{2}, m\right)=S \int_{0}^{+\infty} \frac{d \omega}{2 \pi} \int_{0}^{+\infty} \frac{2 \pi k d k}{(2 \pi)^{2}} \ln f\left(i \omega, k, l_{1}, a, l_{2}, m\right)
$$

where

$$
\begin{aligned}
f\left(i \omega, k, l_{1}, a, l_{2}, m\right) & =1-r_{\text {down }}\left(i \omega, k, l_{1}, m\right) r_{\text {up }}\left(i \omega, k, a, l_{2}, m\right) \\
r_{\text {down }}\left(i \omega, k, l_{1}, m\right) & =\frac{\left(\rho_{2}+\rho_{1}\right)\left(\rho_{3}-\rho_{2}\right)+\left(\rho_{2}-\rho_{1}\right)\left(\rho_{3}+\rho_{2}\right) e^{-2 \rho_{2} l_{1}}}{\left(\rho_{2}+\rho_{1}\right)\left(\rho_{3}+\rho_{2}\right)+\left(\rho_{2}-\rho_{1}\right)\left(\rho_{3}-\rho_{2}\right) e^{-2 \rho_{2} l_{1}}} \\
r_{\text {up }}\left(i \omega, k, a, l_{2}, m\right) & =\frac{\left(\rho_{4}+\rho_{5}\right)\left(\rho_{3}-\rho_{4}\right)+\left(\rho_{4}-\rho_{5}\right)\left(\rho_{3}+\rho_{4}\right) e^{-2 \rho_{4} l_{2}}}{\left(\rho_{4}+\rho_{5}\right)\left(\rho_{3}+\rho_{4}\right)+\left(\rho_{4}-\rho_{5}\right)\left(\rho_{3}-\rho_{4}\right) e^{-2 \rho_{4} l_{2}}} e^{-2 \rho_{3} a}
\end{aligned}
$$

with definitions:

$$
\rho_{1}^{2}=\rho_{3}^{3}=\rho_{5}^{2}=k^{2}+\omega^{2}+m^{2}, \quad \rho_{2}^{2}=\rho_{4}^{2}=k^{2}+\omega^{2} .
$$

Here $r_{\text {down }}\left(\omega, k, l_{1}, m\right)$ and $r_{u p}\left(\omega, k, a, l_{2}, m\right)$ are reflection coefficients of the downward and upward going plane waves reflecting at the lower $(z=0)$ and upper $(z=a)$ boundaries of the layer 3 respectively (see [31] for a derivation of formulas analogous to (4.20)). The factor $e^{-2 \rho_{3} a}$ in (4.23) appears due to a mirror translation of the upper boundary from a position $z=0$ to a position $z=a$ or, in other words, after the change of coordinates $z=-z^{\prime}+a$.

It is convenient to switch to polar coordinates: $r^{2}=k^{2}+\omega^{2}$ with $k=r \cos (\theta)$. Then (4.20) can be expressed as

$$
E_{c}=\frac{S}{(2 \pi)^{2}} \int_{0}^{+\infty} r^{2} \ln f\left(r, l_{1}, a, l_{2}, m\right) d r .
$$


The $\rho$ 's are now given by

$$
\rho_{1}=\rho_{3}=\rho_{5}=\sqrt{r^{2}+m^{2}}, \quad \rho_{2}=\rho_{4}=r
$$

and we obtain

$$
f\left(r, l_{1}, a, l_{2}, m\right)=1-r_{\text {down }}\left(r, l_{1}, m\right) r_{u p}\left(r, a, l_{2}, m\right)
$$

where

$$
r_{\text {down }} r_{u p}=\frac{m^{4}\left(1-e^{-2 r \ell_{1}}\right)\left(1-e^{-2 r \ell_{2}}\right) e^{-2 \sqrt{r^{2}+m^{2}} a}}{\left(r+\sqrt{r^{2}+m^{2}}\right)^{4}-m^{4}\left(e^{-2 r \ell_{1}}+e^{-2 r \ell_{2}}\right)+\left(\sqrt{r^{2}+m^{2}}-r\right)^{4} e^{-2 r\left(\ell_{1}+\ell_{2}\right)}} .
$$

We now replace $m$ by $m_{n}=n / R$ in (4.28) and also make the substitution $u=r R$ :

$$
r_{\text {down }} r_{u p}=g\left(n, u, \ell_{1}, \ell_{2}, R\right) e^{-2 \sqrt{u^{2}+n^{2}} a / R}
$$

where

$$
g=\frac{n^{4}\left(1-e^{-2 u \ell_{1} / R}\right)\left(1-e^{-2 u \ell_{2} / R}\right)}{\left(u+\sqrt{u^{2}+n^{2}}\right)^{4}-n^{4}\left(e^{-2 u \ell_{1} / R}+e^{-2 u \ell_{2} / R}\right)+\left(\sqrt{u^{2}+n^{2}}-u\right)^{4} e^{-2 u\left(\ell_{1}+\ell_{2}\right) / R}} .
$$

Note that $g$ is independent of the plate separation $a$. The contribution to the Casimir energy from Proca continuum modes $E_{p r c}$ is given by

$$
E_{p r c}=2 \sum_{n=1}^{\infty} E_{c}\left(l_{1}, a, l_{2}, n / R\right)
$$

where the factor of two includes the contributions of $A_{z}^{(n)}$ and $A_{z}^{(n) *}$. The Casimir pressure from the Proca continuum modes is then given by

$$
\begin{aligned}
P_{p r c}\left(l_{1}, a, l_{2}, R\right) & =-\frac{2}{S} \sum_{n=1}^{\infty} \frac{\partial E_{c}\left(l_{1}, a, l_{2}, n / R\right)}{\partial a} \\
& =-\frac{1}{\pi^{2} R^{4}} \sum_{n=1}^{\infty} \int_{0}^{\infty} u^{2} \sqrt{u^{2}+n^{2}} \frac{g e^{-2 \sqrt{u^{2}+n^{2}} a / R}}{1-g e^{-2 \sqrt{u^{2}+n^{2}} a / R}} d u .
\end{aligned}
$$

The above formula for the pressure due to the Proca continuum modes converges exponentially fast and is dependent on the thicknesses $\ell_{1}$ and $\ell_{2}$ of the perfect conductors. In Fig. $3, P_{\text {prc }}$ is plotted as a function of the circumference $d=2 \pi R$ and the thickness of the two slabs. In Fig. 3 , lengths are expressed in units of the plate separation $a, P_{p r c}$ is in units of Casimir's result $-\pi^{2} /\left(240 a^{4}\right)$ and the two slabs are assumed to have equal thickness $\left(\ell_{1}=\ell_{2}\right)$. The pressure $P_{\text {prc }}$ increases as the circumference $d$ of the compact dimension increases and as the thickness $\ell_{1}$ of the conductors increases. In the limit $d \rightarrow 0(R \rightarrow 0)$, the pressure $P_{\text {prc }}$ tends to zero 
exponentially fast regardless of the thickness of the slabs. Conversely, in the infinitely thin limit of the conductors where $\ell_{1} \rightarrow 0, P_{p r c}$ tends to zero regardless of the value of $R$. For $d=2, P_{\text {prc }}$ reaches a maximum of approximately $0.6 \%$ of Casimir's result (the maximum value is reached in the $\ell_{1} \rightarrow \infty$ limit. This is close to the $\ell_{1}=10$ result in Fig. 3). For a given $d$, the maximum value of $P_{p r c}$ is significantly less than the contribution $P_{p r d}$ from the Proca discrete modes (plotted in Fig. 2). For example, at $d=2, P_{\text {prd }}$ makes a $12.1 \%$ contribution while $P_{\text {prc }}$ makes a maximum contribution of only $0.6 \%$. Hence, the bulk of the correction to Casimir's result due to the presence of the compact dimension stems from the Proca discrete modes.

The total Casimir pressure $P$ on the plates is obtained by summing the contribution $P_{0}$ from the massless sector and the contribution $P_{p r d}+P_{p r c}$ from the Proca sector:

$$
\begin{aligned}
P= & P_{0}+P_{p r d}+P_{p r c}= \\
= & -\frac{\pi^{2}}{240 a^{4}}-\sum_{n=1}^{\infty} \sum_{\ell=1}^{\infty}\left[\frac{3}{2} \frac{n^{2}}{\pi^{2} R^{2} a^{2} \ell^{2}} K_{2}(2 \ell n a / R)+\frac{n^{3}}{\ell \pi^{2} R^{3} a} K_{1}(2 \ell n a / R)\right] \\
& -\frac{1}{\pi^{2} R^{4}} \sum_{n=1}^{\infty} \int_{0}^{\infty} u^{2} \sqrt{u^{2}+n^{2}} \frac{g e^{-2 \sqrt{u^{2}+n^{2}} a / R}}{1-g e^{-2 \sqrt{u^{2}+n^{2}} a / R}} d u
\end{aligned}
$$

where $g$ is given by (4.30). Equation (4.34) is our final result. Both $P_{p r d}$ and $P_{p r c}$ tend to zero as $R \rightarrow 0$ and one recovers Casimir's parallel plate result $P_{0}=-\frac{\pi^{2}}{240 a^{4}}$ in this limit. Since $P_{p r d}$ and $P_{p r c}$ are manifestly negative, the magnitude of the Casimir pressure increases in the presence of the extra compact dimension (it becomes more negative). Our final result (4.34) naturally differs from previous results [8, 9] because the correction to Casimir's result is not derived using three discrete modes but two discrete and one continuum mode. Had we used three instead of two discrete modes the correction from the Proca discrete modes would have been a factor of $3 / 2$ times higher (e.g. $18.2 \%$ instead of $12.1 \%$ for a circumference $d=2$ ). Instead of a third discrete mode, we obtain a continuum mode which is qualitatively and numerically different.

\section{Conclusions}

More than two decades ago, the Casimir effect in the $4 D$ Proca case where the photon has a mass $m$ was studied for perfectly conducting parallel plates [11, 32. Barton and Dombey [11] showed that in the presence of conductors there are two discrete modes and one continuum mode and not three discrete modes. Both the discrete and continuum modes contributed corrections to Casimir's result. They derived the leading continuum mode contribution for a small mass $m$ and found that it vanishes in the limit $m \rightarrow 0$. Casimir's parallel plate result was recovered in the limit $m \rightarrow 0$. 
The $5 D$ Maxwell problem with one dimension compactified to a circle is closely related to the Proca problem. As in the Proca case, the polarizations in the presence of conducting plates yield discrete and continuum modes. The existence of continuum modes which propagate inside the perfect conductors is a qualitative distinction between the $5 D\left(M^{4} \times S^{1}\right)$ and the $4 D$ Maxwell problem. The Casimir force in the $5 D$ spacetime depends on the thicknesses of the slabs even for perfectly conducting boundary conditions, which is an interesting manifestation of the extra compact dimension. We derive exact results for the contributions of both the discrete and continuum modes and our final result for the Casimir pressure on the plates is given by (4.34). The corrections to Casimir's result are manifestly negative and increase the magnitude of the Casimir pressure on the plates. We plot the corrections versus the circumference $d=2 \pi R$ for the discrete and continuum modes. For the case $d=2$ (in units of the plate separation) the correction is $12.1 \%$ for the discrete modes and a maximum of $0.6 \%$ for the continuum modes. Both contributions decrease exponentially fast as $d$ decreases and Casimir's parallel plate result is recovered in the limit as the radius $R$ of the compact dimension tends to zero.

The parallel plate geometry is a natural theoretical bench-mark for calculating new effects such as those originating from extra compact dimensions. Casimir experiments involving parallel plates are notoriously difficult and the most precise experiments to date have reached only $15 \%$ precision [33]. Measurements have also been carried out to a precision of $1 \%$ for sphere-plate separations in the range $0.1-0.9 \mu \mathrm{m}$ in another set of experiments 34. The experiments involving a micromachined torsional oscillator now lead to a precision better than $1 \%$ [35. In these experiments one measures a gradient of the Casimir force between a sphere and a plate. The gradient of the force between the sphere and the plate can be expressed for a wide range of distances in terms of the force between the two plates $F_{p p}: F_{P S}^{\prime}=2 \pi R_{S} F_{P P}$, where $R_{S}$ is the radius of the sphere. Thus the theoretical results for the Casimir force between the two plates can be verified with a remarkable precision.

\section{A Manifestly negative expression for the Dirichlet Casimir pis- ton in $d+1$-dimensions}

Consider a $d$-dimensional rectangular resonator $R=[0, a] \times M$, where $M$ is a $d$-1-dimensional Dirichlet resonator with a boundary $\partial M$. Eigenfrequencies of the resonator $R$ with Dirichlet boundary conditions are determined by

$$
\begin{aligned}
& \omega^{2}=\left(\frac{\pi l}{a}\right)^{2}+\lambda_{k D}^{2}, \quad l=1 . .+\infty, k=1 . .+\infty \\
& \Delta^{(d-1)} f_{k}(x, y)=-\lambda_{k D}^{2} f_{k}(x, y) \\
& \left.f_{k}(x, y)\right|_{\partial M}=0,
\end{aligned}
$$


$\Delta^{(d-1)}$ is a $d-1$-dimensional Laplace operator. One can write $\lambda_{k D}$ explicitly:

$$
\lambda_{k D}=\sqrt{\sum_{i=1}^{d-1}\left(\frac{\pi k_{i}}{L_{i}}\right)^{2}},
$$

here $L_{i}$ are lengths of the sides of the resonator $M, k_{i}$ are positive integers.

We adopt the zeta function regularization [36, 37]. The Casimir energy is defined then as follows:

$$
E=\left.\sum \frac{\omega^{1-s}}{2}\right|_{s=0}
$$

This sum has to be evaluated for large positive values of $s$, an analytical continuation to the value $s=0$ is being performed at the end of calculations.

Alternatively one can define the Casimir energy via a zero temperature one loop effective action $W$ [38] ( $T_{1}$ is a time interval here):

$$
\begin{aligned}
W & =E T_{1} \\
E & =-\zeta^{\prime}(0) \\
\zeta(s) & =\frac{1}{\Gamma\left(\frac{s}{2}\right)} \int_{0}^{+\infty} d t t^{\frac{s}{2}-1} \sum_{\omega} \int_{-\infty}^{+\infty} \frac{d p}{2 \pi} \exp \left(-t\left(\frac{a}{\pi}\right)^{2}\left(\omega^{2}+p^{2}\right)\right)
\end{aligned}
$$

After integration over $p$ in (A.8) one can verify that definitions (A.5) and (A.7) coincide.

In every Casimir sum it is convenient to write:

$$
\sum_{l=1}^{+\infty} \exp \left(-t l^{2}\right)=\frac{1}{2} \theta_{3}\left(0, \frac{t}{\pi}\right)-\frac{1}{2} .
$$

For the first term on the right-hand side of (A.9) we use the property of the theta function $\theta_{3}(0, x)$ :

$$
\theta_{3}(0, x)=\frac{1}{\sqrt{x}} \theta_{3}\left(0, \frac{1}{x}\right)
$$

and the value of the integral

$$
\int_{0}^{+\infty} d t t^{\alpha-1} \exp \left(-p t-\frac{q}{t}\right)=2\left(\frac{q}{p}\right)^{\frac{\alpha}{2}} K_{\alpha}(2 \sqrt{p q})
$$

expressed in terms of a modified Bessel function $K_{\alpha}(x)$ for nonzero values of $n$ to rewrite the 
zeta function $\zeta(s)$ in the form:

$$
\begin{aligned}
\zeta(s)=\sum_{\lambda_{k D}} \int_{-\infty}^{+\infty} \frac{d p}{2 \pi}\left[\frac{\sqrt{\pi} \Gamma((s-1) / 2)}{2 \Gamma(s / 2)}\left(\frac{a \sqrt{\lambda_{k D}^{2}+p^{2}}}{\pi}\right)^{1-s}\right. \\
\left.+\sum_{l=1}^{+\infty} \frac{2 \sqrt{\pi}}{\Gamma(s / 2)}\left(\frac{\pi^{2} l}{a \sqrt{\lambda_{k D}^{2}+p^{2}}}\right)^{\frac{s-1}{2}} K_{\frac{s-1}{2}}\left(2 a l \sqrt{\lambda_{k D}^{2}+p^{2}}\right)\right] \\
+\sum_{\lambda_{k D}} \frac{\sqrt{\pi} \Gamma((s-1) / 2)}{4 a \Gamma(s / 2)}\left(\frac{a \lambda_{k D}}{\pi}\right)^{1-s}
\end{aligned}
$$

The Casimir energy of a resonator $R$ is given by:

$$
\begin{aligned}
E=\sum_{\lambda_{k D}} \int_{-\infty}^{+\infty} \frac{d p}{2 \pi} \frac{1}{2} \ln \left(1-\exp \left(-2 a \sqrt{\lambda_{k D}^{2}+p^{2}}\right)\right) \\
+\left.\frac{a}{2} \sum_{\lambda_{k D}} \int_{-\infty}^{+\infty} \frac{d p}{2 \pi}\left(\lambda_{k D}^{2}+p^{2}\right)^{\frac{1-s}{2}}\right|_{s=0}+\left.\frac{1}{4} \sum_{\lambda_{k D}} \lambda_{k D}^{1-s}\right|_{s=0} .
\end{aligned}
$$

Here we used the property $K_{-1 / 2}(x)=\sqrt{\pi /(2 x)} \exp (-x)$.

The term

$$
E_{\text {cylinder }}=\left.\frac{1}{2} \sum_{\lambda_{k D}} \int_{-\infty}^{+\infty} \frac{d p}{2 \pi}\left(\lambda_{k D}^{2}+p^{2}\right)^{\frac{1-s}{2}}\right|_{s=0}
$$

can be thought of as the Casimir energy per unit length of an infinite cylinder with Dirichlet boundary conditions and the same $d$-1-dimensional section $M$ as the resonator $R$.

For the experimental check of the Casimir energy one should measure the force. One can insert two $d$-1-dimensional plates $M$ inside an infinite cylinder with the same $d$-1-dimensional cross section $M$ and measure the force acting on one of the plates as it is being moved through the cylinder. The distance between the plates is $a$.

To calculate the force on each of the two plates inside a cylinder with the cross section $M$ one can perform the following gedanken experiment that was frequently used to calculate the Casimir force between two infinite parallel plates. Imagine that 4 parallel plates are inserted inside an infinite cylinder and then 2 exterior plates are moved to spatial infinity. This situation is exactly equivalent to 3 cavities touching each other. From the energy of this system one has to subtract the Casimir energy of an infinite cylinder without plates inside it, only then do we obtain the energy of interaction between the interior parallel plates, the one that can be measured in the experiment. The force on each of the interior parallel plates is the same 
as the force on the piston when one of the three piston plates (the exterior plate) is moved to infinity. So the attractive force on each of the $d$-1-dimensional Dirichlet pistons inside the $d$-dimensional Dirichlet cylinder is equal to:

$$
F(a)=-\frac{\partial \mathcal{E}(a)}{\partial a}
$$

where the Casimir energy of a $d$-1-dimensional piston with Dirichlet boundary conditions can be written as follows:

$$
\begin{aligned}
\mathcal{E}(a) & =\sum_{\omega_{c}} \frac{1}{2} \ln \left(1-\exp \left(-2 a \omega_{c}\right)\right)= \\
& =\sum_{\lambda_{k D}} \int_{-\infty}^{+\infty} \frac{d p}{2 \pi} \frac{1}{2} \ln \left(1-\exp \left(-2 a \sqrt{\lambda_{k D}^{2}+p^{2}}\right)\right),
\end{aligned}
$$

the sum here is over all eigenfrequencies $\omega_{c}$ for a cylinder with a $d$-1-dimensional cross section $M$ and an infinite length with Dirichlet boundary conditions imposed.

By making use of an identity [39]

$$
\frac{1}{2} \int_{-\infty}^{+\infty} \frac{d p}{2 \pi} \ln \left(1-\exp \left(-2 a \sqrt{\lambda^{2}+p^{2}}\right)\right)=-\frac{\lambda}{2 \pi} \sum_{l=1}^{+\infty} \frac{K_{1}(2 l \lambda a)}{l}
$$

one can rewrite (A.16) in the form:

$$
\begin{aligned}
\mathcal{E}(a) & =-\frac{1}{2 \pi} \sum_{l=1}^{+\infty} \sum_{\lambda_{k D}} \frac{\lambda_{k D} K_{1}\left(2 l \lambda_{k D} a\right)}{l}, \\
\lambda_{k D} & =\sqrt{\sum_{i=1}^{d-1}\left(\frac{\pi k_{i}}{L_{i}}\right)^{2}}
\end{aligned}
$$

the sum should be performed over all sets of positive integer values of $k_{i}$.

\section{Acknowledgments}

AE acknowledges support from a discovery grant of the Natural Sciences and Engineering Research Council of Canada (NSERC). VM acknowledges support from a CNRS grant ANR06-NANO-062 and grants RNP 2.1.1.1112, SS 5538.2006.2 and RFBR 07-01-00692-a. AE would like to thank the Kavli Institute for Theoretical Physics (KITP, Santa Barbara) for their hospitality and financial support where part of this work was completed and presented (this research was supported in part by the National Science Foundation under Grant No. PHY05-51164). AE would also like to thank professor Raman Sundrum of John Hopkins University for email correspondence. 


\section{References}

[1] T. Kaluza, Preus. Acad. Wiss. K1, 966 (1921) ;

[2] O. Klein, Z. Phys. 37, 895 (1926).

[3] B.R. Greene and J. Levin, JHEP 096 (2007) 0711 arXiv:0707.1062.

[4] A.A. Saharian and M.R. Setare, Phys. Lett. B 659, 367 (2008) arXiv:0707.3240]; A.A. Saharian and M.R. Setare, Phys. Lett. B 552, 119 (2003) [hep-th/0207138].

[5] M. Frank, N. Saad and I. Turin, arXiv:0807.0443.

[6] R. Obousy and G. Cleaver, arXiv:0810.1096].

[7] H. Cheng, arXiv:0801.2810.

[8] L. Perivolaropoulos, Phys. Rev. D 77, 107301 (2008) arXiv:0802.1531].

[9] K. Poppenhaeger, S. Hossenfelder, S. Hofmann and M. Bleicher, Phys. Lett. B 582, 1 (2004).

[10] R. Sundrum, TASI lectures hep-th/0508134.

[11] G. Barton and N. Dombey, Nature 311, 336, (1984); G. Barton and N. Dombey, Ann. of Phys. 162, 231 (1985).

[12] E. M. Lifshitz, Zh.Eksp.Teor.Fiz. 29, 94 (1955) ; Soviet Phys. JETP 2, 73 (1956).

[13] H. B. G. Casimir, Proc. Kon. N. Akad. Wet. 51, 793 (1948).

[14] V.N. Marachevsky, Phys. Rev. D 75, 085019 (2007) hep-th/0703158.

[15] R. M. Cavalcanti, Phys. Rev. D 69, 065015 (2004).

[16] A. Edery, Phys. Rev. D 75, 105012 (2007) hep-th/0610173]; J. Phys. A: Math. Gen. 39, 685 (2006) math-ph/0510056.

[17] M.P. Hertzberg, R.L. Jaffe, M. Kardar and A. Scardicchio, Phys. Rev. Lett.95, 250402 (2005) quant-ph/0509071; Phys. Rev. D 76, 045016 (2007) arXiv:0705.0139.

[18] A. Edery and V. Marachevsky, Phys. Rev. D 78 , 025021 (2008). arXiv:0805.4038.

[19] V.N. Marachevsky, J. Phys. A: Math. Theor. 41, 164007 (2008). arXiv:0710.4130].

[20] A. Edery and I. MacDonald, JHEP 09, 005 (2007) arXiv:0708.0392. 
[21] V.N. Marachevsky, hep-th/0609116, published in the proceedings of QUARKS-2006.

[22] V.N. Marachevsky, hep-th/0512221.

[23] G. Barton, Phys. Rev. D 73, 065018 (2006).

[24] A. Edery, J. Stat. Mech. P06007 (2006) hep-th/0510238.

[25] S.A. Fulling, L. Kaplan and J.H. Wilson, Phys. Rev. A 76, 012118 (2007) [arXiv:0703248].

[26] S. C. Lim and L.P. Teo, arXiv:0807.3613.

[27] S. C. Lim and L.P. Teo, arXiv:0808.0047].

[28] Yu.S. Barash and V.L. Ginzburg, Sov.Phys.Usp. 18, 305 (1975).

[29] K.A. Milton, J.Phys.A: Math. Gen. 37, R209 (2004).

[30] S.A. Ellingsen and I. Brevik, J.Phys.A: Math.Gen. 40, 3643 (2007).

[31] A. Lambrecht and V.N. Marachevsky, Phys. Rev. Lett. 101, 160403 (2008) [arXiv: 0806.3142].

[32] P.C.W. Davies and S. Unwin, Phys. Lett. B 98 274, (1981).

[33] G. Bressi, G. Carugno, R. Onofrio and G. Ruoso, Phys. Rev. Lett. 88, 041804 (2002).

[34] U. Mohideen and A. Roy, Phys. Rev. Lett. 81,4549 (1998); A. Roy, C.Lin and U. Mohideen, Phys. Rev. D 60,111101 (1999); M.Bordag, U.Mohideen and V.M.Mostepanenko, Phys.Rep. 353, 1 (2001).

[35] R.S. Decca, D. Lopez, E. Fischbach, G.L. Klimchitskaya, D.E. Krause, V.M. Mostepanenko, Ann.Phys. 318, 37 (2005); Eur.Phys.J.C 51, 963 (2007).

[36] E.Elizalde, Ten physical applications of spectral zeta functions (Springer) (1995).

[37] K.Kirsten, Spectral functions in Mathematics and Physics (Boca Raton, FL: CRC Press) (2002).

[38] D.V. Vassilevich, Phys.Rep. 388, 279 (2003).

[39] V.V. Nesterenko and I.G. Pirozhenko, J.Math.Phys.38, 6265 (1997) [arXiv: hep-th/9703097]. 


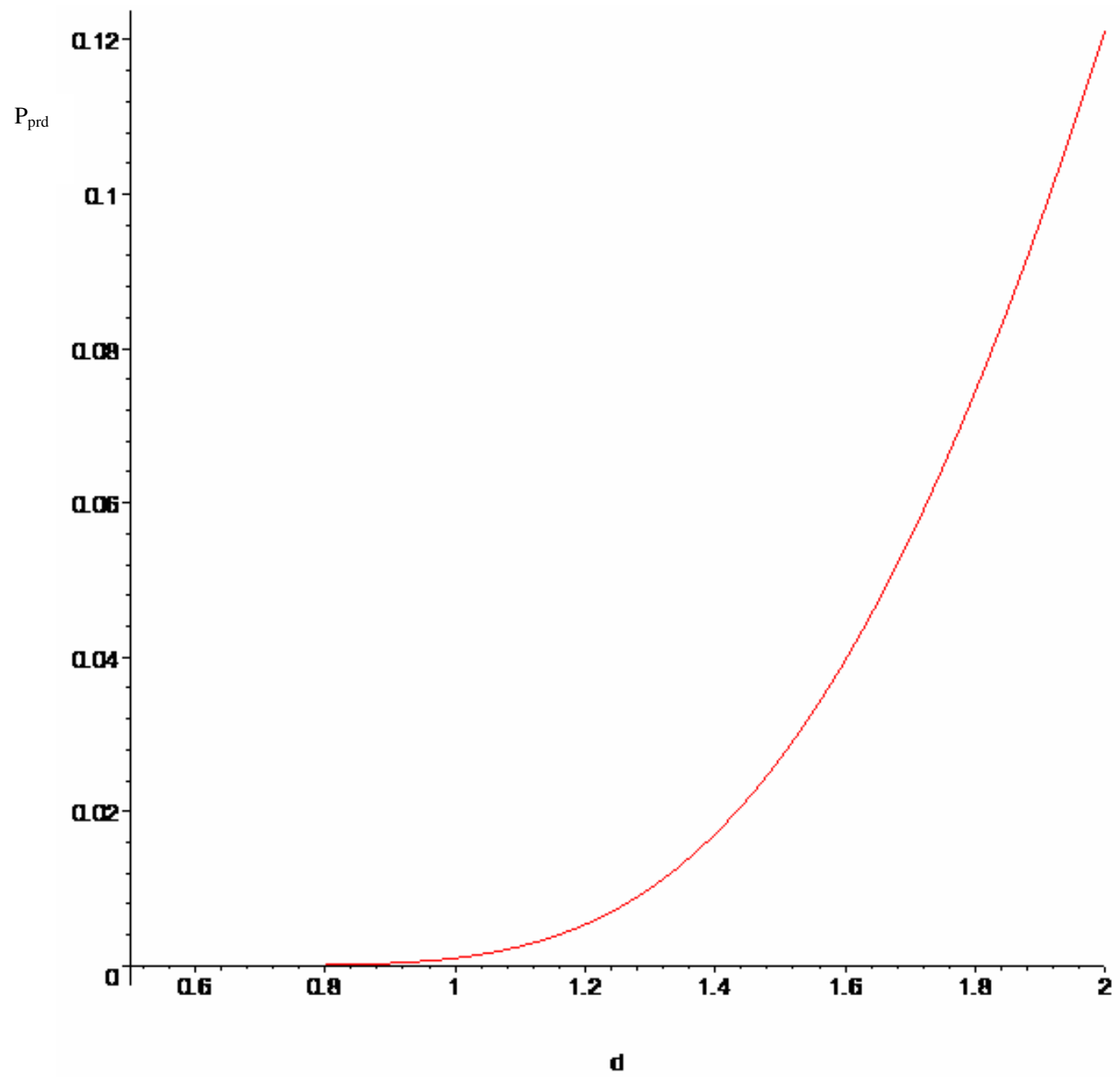

Figure 2: Correction from Proca discrete modes $P_{p r d}$ (in units of Casimir's parallel plate result) as a function of the circumference $d$ of the compact dimension (in units of the plate separation a) 


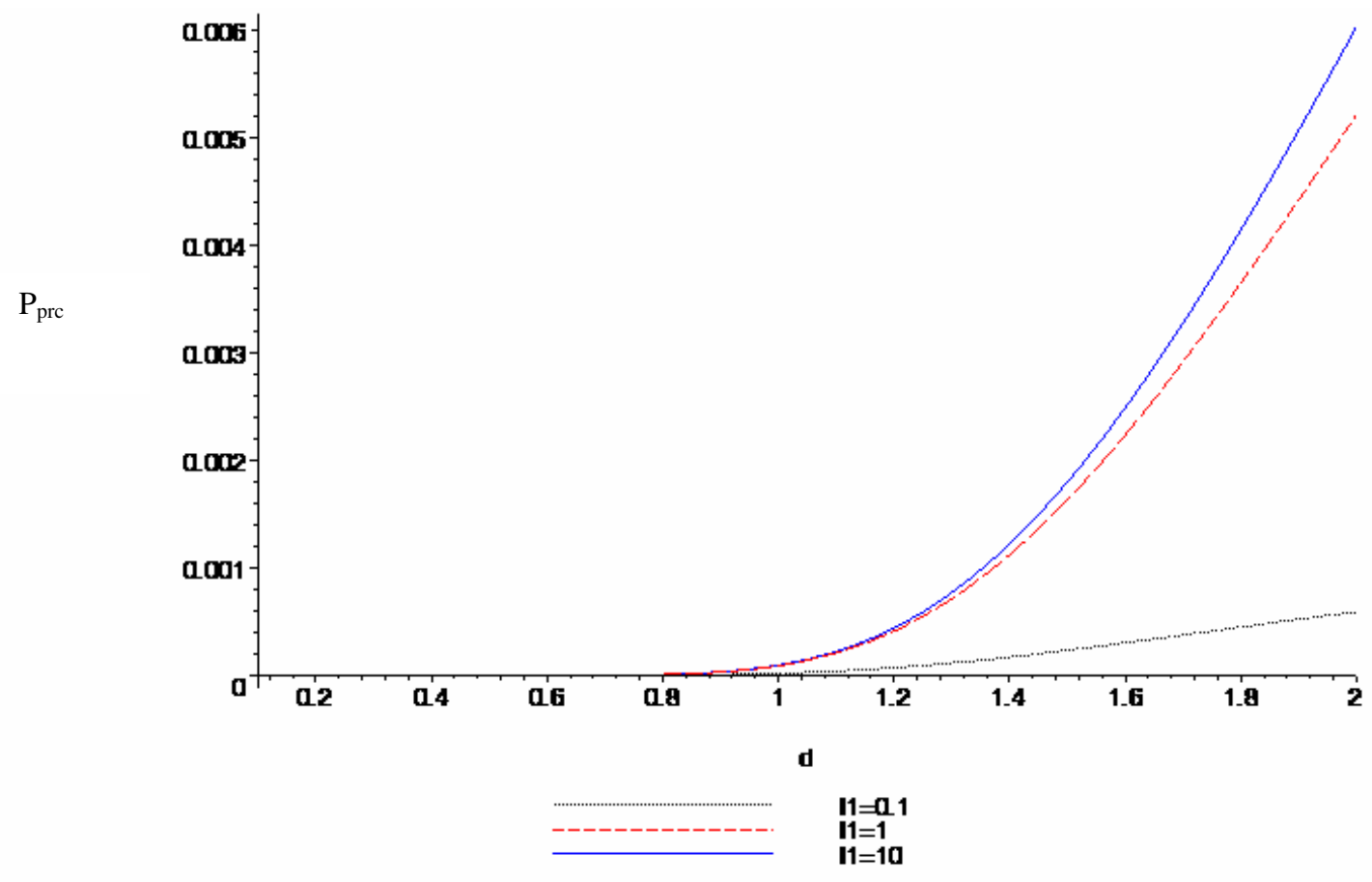

Figure 3: Correction from the Proca continuum mode $P_{p r c}$ (in units of Casimir's parallel plate result) as a function of the circumference $d$ of the compact dimension and the thickness $\ell_{1}$ of the two slabs ( $d$ and $\ell_{1}$ are in units of the plate separation $a$ ). 